\title{
On Harmonious Labeling of Corona Graphs
}

\author{
Martin Bača ${ }^{1}$ and Maged Z. Youssef ${ }^{2,3}$ \\ ${ }^{1}$ Department of Applied Mathematics and Informatics, Technical University, 04200 Košice, Slovakia \\ ${ }^{2}$ Department of Mathematics and Statistics, College of Sciences, Al-Imam Mohammed Ibn Saud Islamic University, \\ Riyadh 11623, Saudi Arabia \\ ${ }^{3}$ Department of Mathematics, Faculty of Science, Ain Shams University, Abbassia, Cairo 11566, Egypt
}

Correspondence should be addressed to Martin Bača; martin.baca@tuke.sk

Received 9 January 2014; Accepted 12 March 2014; Published 3 April 2014

Academic Editor: Hui-Shen Shen

Copyright (c) 2014 M. Bača and M. Z. Youssef. This is an open access article distributed under the Creative Commons Attribution License, which permits unrestricted use, distribution, and reproduction in any medium, provided the original work is properly cited.

A graph $G$ with $q$ edges is said to be harmonious, if there is an injection $f$ from the vertices of $G$ to the group of integers modulo $q$ such that when each edge $x y$ is assigned the label $f(x)+f(y)(\bmod q)$, the resulting edge labels are distinct. In this paper, we study the existence of harmonious labeling for the corona graphs of a cycle and a graph $G$ and for the corona graph of $K_{2}$ and a tree.

\section{Introduction}

Harmonious graphs naturally arose in the study of modular version of error-correcting codes and channel assignment problems. Graham and Sloane [1] defined a $(p, q)$-graph $G$ of order $p$ and size $q$ to be harmonious, if there is an injective function $f: V(G) \rightarrow \mathbb{Z}_{q}$, where $\mathbb{Z}_{q}$ is the group of integers modulo $q$, such that the induced function $f^{*}: E(G) \rightarrow \mathbb{Z}_{q}$, defined by $f^{*}(x y)=f(x)+f(y)$ for each edge $x y \in E(G)$, is a bijection.

The function $f$ is called harmonious labeling and the image of $f$ denoted by $\operatorname{Im}(f)$ is called the corresponding set of vertex labels.

When $G$ is a tree or, in general for a graph $G$ with $p=q+1$, exactly one label may be used on two vertices.

Graham and Sloane [1] proved that if a harmonious graph has an even number of edges $q$ and the degree of every vertex is divisible by $2^{k}$, then $q$ is divisible by $2^{k+1}$. This necessary condition is called the harmonious parity condition. They also proved that if $f$ is harmonious labeling of a graph $G$ of size $q$, then so is $a f+b$ labeling, where $a$ is an invertible element of $\mathbb{Z}_{q}$ and $b$ is any element of $\mathbb{Z}_{q}$

Chang et al. [2] define an injective labeling $f$ of a graph $G$ with $q$ edges to be strongly c-harmonious, if the vertex labels are from the set $\{0,1, \ldots, q-1\}$ and the edge labels are from the set $\left\{f^{*}(x y)=f(x)+f(y): x y \in E(G)\right\}=\{c, c+1, \ldots, c+$ $q-1\}$. Grace $[3,4]$ called such labeling sequential. In the case of a tree, Grace allows the vertex labels to range from 0 up to $q$. Strongly 1-harmonious graph is called strongly harmonious.

By taking the edge labels of a sequentially labeled graph with $q$ edges modulo $q$, we obviously obtain a harmoniously labeled graph. It is not known if there is a graph that can be harmoniously labeled but not sequentially labeled. More than 50 papers have been published on harmonious labeling and comprehensive information can be found in [5]. Similarly, labeling of special types of crown graphs is examined in [6].

In this paper, we study the existence of harmonious labeling for the graphs obtained by corona operation between a cycle and a graph $G$ and also between $K_{2}$ and a tree or $K_{2}$ and a unicyclic graph.

\section{Main Results}

In this section, we present the results related to corona graphs. The corona operation between two graphs was introduced by Frucht and Harary [7]. Given two graphs $G$ of order $p$ and $H$, the corona of $G$ with $H$, denoted by $G \odot H$, is the graph with $V(G \odot H)=V(G) \cup \bigcup_{i=1}^{p} V\left(H_{i}\right)$, and $E(G \odot H)=E(G) \cup$ $\bigcup_{i=1}^{p}\left(E\left(H_{i}\right) \cup\left\{\left(v_{i}, u\right): v_{i} \in V(G)\right.\right.$ and $\left.\left.u \in V\left(H_{i}\right)\right\}\right)$. In other words, a corona graph is obtained from two graphs, $G$ of order $p$ and $H$, taking one copy of $G$ and $p$ copies of $H$ and joining by an edge the $i$ th vertex of $G$ to every vertex in the $i$ th copy of $H$. 
Grace [4] showed that $C_{2 n+1} \odot K_{1}$ is harmonious and conjectured that $C_{2 n} \odot K_{1}$ is harmonious. This conjecture has been proved by Liu and Zhang [8] and Liu [9]. Singh in [10, 11] has proved that $C_{n} \odot K_{2}$ and $C_{n} \odot K_{3}$ are sequential for all odd $n>1$. Santhosh [12] has shown that $C_{n} \odot P_{4}$ is sequential for all odd $n \geq 3$.

The join of two graphs $G$ and $H$, denoted by $G+H$, is the graph where $V(G) \cap V(H)=\emptyset$ and each vertex of $G$ is adjacent to all vertices of $H$. When $H=K_{1}$, this is the corona graph $K_{1} \odot G$.

Graham and Sloane [1] showed harmonious labeling of the join of the path $P_{n}$ and $K_{1}$, that is, the fan $F_{n}=P_{n}+K_{1}$, and harmonious labeling of the double fan $P_{n}+\overline{K_{2}}$. Later, Chang et al. [2] gave harmonious labeling of the join of the star $S_{n}$ and $K_{1}$.

The next result shows that if join of a graph $G$ and $K_{1}$ is strongly harmonious, then the corona of a cycle and the graph $G$ admitted harmonious labeling.

Theorem 1. Let $G$ be a graph of order $p$ and size q. If $G+K_{1}$ is strongly harmonious with the 0 label on the vertex of $K_{1}$, then $C_{n} \odot G$ is harmonious for all odd $n \geq 3$.

Proof. Let $G$ be a $(p, q)$-graph and $G+K_{1}$ strongly harmonious with the 0 label on the vertex $x \in K_{1}$. Then, there exists labeling $f: V\left(G+K_{1}\right) \rightarrow\{0, \ldots, p+q-1\}$ such that $f(x)=0$ and the edge labels are from the set $\left\{f^{*}(u v)=f(u)+f(v)\right.$ : $\left.u v \in E\left(G+K_{1}\right)\right\}=\{1,2, \ldots, p+q\}$.

Now, for $n$ odd, $n \geq 3$, we consider the corona graph $C_{n} \odot G$ with $n(p+1)$ vertices and $\Gamma=n(p+q+1)$ edges. Denote the vertices and edges of the cycle $C_{n}$ such that $V\left(C_{n}\right)=$ $\left\{x_{1}, x_{2}, \ldots, x_{n}\right\}$ and $E\left(C_{n}\right)=\left\{x_{i} x_{i+1}: 1 \leq i \leq n-1\right\} \cup\left\{x_{n} x_{1}\right\}$. By the symbol $y^{i}$, we denote a vertex in the $i$ th copy of $G$, denoted by $G_{i}$, corresponding to the vertex $y$ in $G$; that is, $y \in V(G)$ and $y^{i} \in V\left(G_{i}\right)$.

We define the vertex labeling $g: V\left(C_{n} \odot G\right) \rightarrow\{0,1, \ldots$, $\Gamma-1\}$ in the following:

$$
\begin{aligned}
& g\left(x_{i}\right)=(p+q+1)(i-1), \quad \text { for } 1 \leq i \leq n, \\
& g\left(y^{i}\right)=f(y)+(p+q+1)(i-1), \quad \text { for } 1 \leq i \leq n .
\end{aligned}
$$

If we denote the join graph $G+K_{1}$ as $G+\{x\}$, then the set of all edge labels of the $i$ th copy of $G+\{x\}$ consists of the consecutive integers $g^{*}\left(E\left(G_{i}+\left\{x_{i}\right\}\right)\right)=\{2(p+q+1)(i-1)+$ $1,2(p+q+1)(i-1)+2, \ldots, 2(p+q+1)(i-1)+p+q\}$, $1 \leq i \leq n$. For edge labels of the cycle $C_{n}$, we have $g^{*}\left(x_{i} x_{i+1}\right)=$ $g\left(x_{i}\right)+g\left(x_{i+1}\right)=(p+q+1)(2 i-1)$, for $1 \leq i \leq n-1$, and $g^{*}\left(x_{n} x_{1}\right)=g\left(x_{n}\right)+g\left(x_{1}\right)=(p+q+1)(n-1)$. that

It is not difficult to see that, for $1 \leq i \leq(n-1) / 2$, it is true

(i) $1+\max \left\{g^{*}\left(E\left(G_{i}+\left\{x_{i}\right\}\right)\right)\right\}=(p+q+1)(2 i-1)=$ $g^{*}\left(x_{i} x_{i+1}\right)$

(ii) $1+g^{*}\left(x_{i} x_{i+1}\right)=\min \left\{g^{*}\left(E\left(G_{((n+1) / 2)+i}+\right.\right.\right.$ $\left.\left.\left.\left\{x_{((n+1) / 2)+i}\right\}\right)\right)\right\}=(p+q+1)(2 i-1)+1(\bmod$ $\Gamma)$;

(iii) $1+\max \left\{g^{*}\left(E\left(G_{((n+1) / 2)+i}+\left\{x_{((n+1) / 2)+i}\right\}\right)\right)\right\}=$ $2(p+q+1) i(\bmod \Gamma)$ and it is equal to $g^{*}\left(x_{((n+1) / 2)+i} x_{((n+1) / 2)+i+1}\right)(\bmod \Gamma)$; (iv) $1+g^{*}\left(x_{((n+1) / 2)+i} x_{((n+1) / 2)+i+1}\right)(\bmod \Gamma)$ is equal to the $\min \left\{g^{*}\left(E\left(G_{i+1}+\left\{x_{i+1}\right\}\right)\right)\right\}=2(p+q+1) i+1$.

Moreover, $1+\max \left\{g^{*}\left(E\left(G_{(n+1) / 2}+\left\{x_{(n+1) / 2}\right\}\right)\right)\right\}=0(\bmod \Gamma)$ and it is equal to $g^{*}\left(x_{(n+1) / 2} x_{(n+3) / 2}\right)=0(\bmod \Gamma)$.

Thus, under the induced mapping $g^{*}$, all the resulting edge labels are distinct and they get the consecutive integers from 0 up to $n(p+q+1)-1(\bmod \Gamma)$. This concludes the proøfraham and Sloane [1] have proved that the fans $F_{m} \square$ $P_{m}+K_{1}, m \leq 7$, and the wheels $W_{m}=C_{m}+K_{1}, m \neq \equiv 2(\bmod$ $3)$, are strongly harmonious with the 0 label on the vertex of $K_{1}$. In light of these results and Theorem 1 , we have the following corollaries.

Corollary 2. Let $C_{n} \odot P_{m}$ be the corona graph of a cycle $C_{n}$ and a path $P_{m}$. Then, $C_{n} \odot P_{m}$ is harmonious for all odd $n \geq 3$ and $1 \leq m \leq 7$

Corollary 3. Let $C_{n} \odot C_{m}$ be the corona graph of two cycles. Then, $C_{n} \odot C_{m}$ is harmonious for all odd $n \geq 3$ and $m \neq \equiv$ $(\bmod 3)$.

Shee [13] has shown that the complete tripartite graph $K_{1, m, k}=K_{m, k}+K_{1}, m, k \geq 1$, is strongly harmonious, while Gnanajothi [14] proved that $K_{1,1, m, k}=K_{1, m, k}+K_{1}, m, k \geq 1$, is also strongly harmonious. In both cases, the vertex of $K_{1}$ is labeled by the 0 label. Thus, with respect to Theorem 1 , we obtain the following.

Corollary 4. For $m, k \geq 1$ and odd $n \geq 3$, the corona graph $C_{n} \odot K_{m, k}$ is harmonious.

Corollary 5. For $m, k \geq 1$ and odd $n \geq 3$, the corona graph $C_{n} \odot K_{1, m, k}$ is harmonious.

Let one consider the graphs obtained by corona operation between the single edge $K_{2}$ and a tree.

Theorem 6. If $T$ is a strongly c-harmonious tree of odd size $q$ and $c=(q+1) / 2$, then the corona graph $K_{2} \odot T$ is also strongly c-harmonious.

Proof. Let $T$ be a tree of size $q$ with strongly $c$-harmonious labeling $f: V(T) \rightarrow\{0,1, \ldots, q\}$, where the edge labels are from the set of consecutive integers $\left\{f^{*}(e): e \in E(T)\right\}=$ $\{c, c+1, \ldots, c+q-1\}$.

Consider the corona graph $K_{2} \odot T$ with vertices $x_{1}, x_{2} \epsilon$ $V\left(K_{2}\right)$ and vertices $y^{i} \in V\left(T_{i}\right), i=1,2$, corresponding to the vertices $y \in T$, where the vertex $x_{i}$ is incident to every vertex in $T_{i}$ for $i=1,2$.

Define now new vertex labeling $g: V\left(K_{2} \odot T\right) \rightarrow$ $\{0,1, \ldots, 4 q+2\}$ such that

$$
g\left(x_{i}\right)= \begin{cases}c+q, & \text { for } i=1 \\ q+1, & \text { for } i=2\end{cases}
$$

$$
g\left(y^{i}\right)= \begin{cases}f(y), & \text { for } i=1 \text { and every } y \in T \\ f(y)+c+q+1, & \text { for } i=2 \text { and every } y \in T\end{cases}
$$


Thus, $\operatorname{Im}(g)=\{0,1,2, \ldots, q, q+1\} \cup\{c+q, c+q+1, c+q+$ $2, \ldots, c+2 q, c+2 q+1\}$ and, for the edge labels, we have

$$
\begin{gathered}
\left\{g^{*}(e): e \in E\left(T_{1}\right)\right\}=\{c, c+1, c+2, \ldots, c+q-1\}, \\
\left\{g^{*}\left(x_{1} y^{1}\right)=g\left(x_{1}\right)+g\left(y^{1}\right): y^{1} \in V\left(T_{1}\right)\right\} \\
=\{c+q, c+q+1, \ldots, c+2 q\}, \\
g^{*}\left(x_{1} x_{2}\right)=g\left(x_{1}\right)+g\left(x_{2}\right)=c+2 q+1, \\
\left\{g^{*}\left(x_{2} y^{2}\right)=g\left(x_{2}\right)+g\left(y^{2}\right): y^{2} \in V\left(T_{2}\right)\right\} \\
=\{c+2 q+2, c+2 q+3, \ldots, c+3 q+2\}, \\
\left\{g^{*}(e): e \in E\left(T_{2}\right)\right\} \\
=\{3 c+2 q+2,3 c+2 q+3, \ldots, 3 c+3 q+1\} .
\end{gathered}
$$

We can see that edge labels form the set of consecutive integers from $c$ up to $3 c+3 q+1$ if and only if $\max \left\{g^{*}\left(x_{2} y^{2}\right)=\right.$ $\left.g\left(x_{2}\right)+g\left(y^{2}\right): y^{2} \in V\left(T_{2}\right)\right\}+1=\min \left\{g^{*}(e): e \in E\left(T_{2}\right)\right\}$; that is, $c=(q+1) / 2$.

We know that every caterpillar Cat ${ }_{p}$ admits strongly $c$ harmonious labeling. As an illustration, Figure 1 provides an example of the strongly 5 -harmonious labeling of Cat ${ }_{10}$.

As an immediate consequence of Theorem 6, we can state the following corollary.

Corollary 7. Let Cat ${ }_{q+1}$ be a caterpillar of odd size q. If Cat ${ }_{q+1}$ admits strongly $(q+1) / 2$-harmonious labeling, then the corona graph $K_{2} \odot$ Cat $_{q+1}$ also admits strongly $(q+1) / 2$ harmonious labeling.

Theorem 8. Let $G$ be a unicyclic graph of odd size q. If $G$ is a strongly c-harmonious and $c=(q-1) / 2$, then the corona graph $\mathrm{K}_{2} \odot \mathrm{G}$ is also strongly c-harmonious.

Proof. Let $G$ be a connected $(p, q)$-graph containing exactly one cycle. Clearly, $p=q$. Let $f: V(G) \rightarrow\{0,1, \ldots, q-1\}$ be strongly $c$-harmonious labeling with the edge labels from the set of consecutive integers $\left\{f^{*}(e): e \in E(G)\right\}=\{c, c+$ $1, \ldots, c+q-1\}$.

If $x_{1}$ and $x_{2}$ are the vertices of $K_{2}$ and if by the symbol $y^{i}$ we mean a vertex in the $i$ th copy of $G$ corresponding to the vertex $y \in V(G)$, then sets of vertices and edges of the corona graph $K_{2} \odot G$ are as follows: $V\left(K_{2} \odot G\right)=V\left(K_{2}\right) \cup$ $V\left(G_{1}\right) \cup V\left(G_{2}\right), E\left(K_{2} \odot G\right)=\left\{x_{1} x_{2}\right\} \cup E\left(G_{1}\right) \cup\left\{x_{1} y^{1}: y^{1} \in\right.$ $\left.V\left(G_{1}\right)\right\} \cup E\left(G_{2}\right) \cup\left\{x_{2} y^{2}: y^{2} \in V\left(G_{2}\right)\right\}$.

Define new vertex labeling $g: V\left(K_{2} \odot G\right) \rightarrow\{0,1, \ldots, 4 q\}$ in the following:

$$
g\left(x_{i}\right)= \begin{cases}c+q, & \text { for } i=1, \\ q, & \text { for } i=2,\end{cases}
$$

$$
g\left(y^{i}\right)= \begin{cases}f(y), & \text { for } i=1 \text { and every } y \in G \\ f(y)+c+q+1, & \text { for } i=2 \text { and every } y \in G\end{cases}
$$

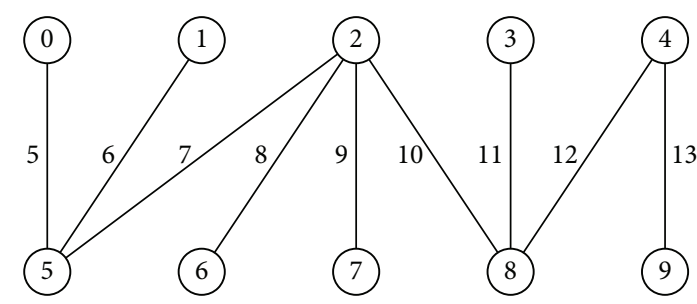

Figure 1: Strongly 5-harmonious labeling of the caterpillar Cat ${ }_{10}$.

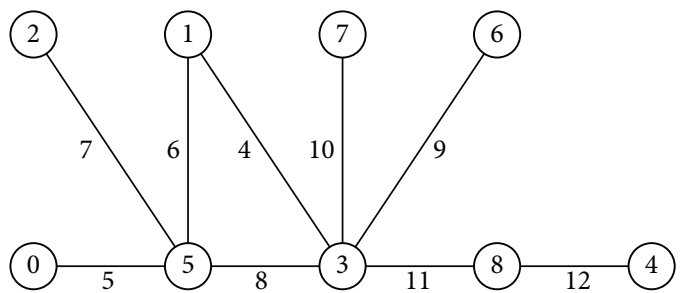

FIGURE 2: Strongly 4-harmonious labeling of a unicyclic graph.

The image of the vertex labeling $g$ is a union of two sets of consecutive integers $\operatorname{Im}(g)=\{0,1,2, \ldots, q\} \cup\{c+q, c+q+$ $1, c+q+2, \ldots, c+2 q\}$. Observe that the edge labels are

$$
\begin{gathered}
\left\{g^{*}(e): e \in E\left(G_{1}\right)\right\}=\{c, c+1, c+2, \ldots, c+q-1\}, \\
\left\{g^{*}\left(x_{1} y^{1}\right)=g\left(x_{1}\right)+g\left(y^{1}\right): y^{1} \in V\left(G_{1}\right)\right\} \\
=\{c+q, c+q+1, \ldots, c+2 q-1\}, \\
g^{*}\left(x_{1} x_{2}\right)=g\left(x_{1}\right)+g\left(x_{2}\right)=c+2 q, \\
\left\{g^{*}\left(x_{2} y^{2}\right)=g\left(x_{2}\right)+g\left(y^{2}\right): y^{2} \in V\left(T_{2}\right)\right\} \\
=\{c+2 q+1, c+2 q+2, \ldots, c+3 q\}, \\
\left\{g^{*}(e): e \in E\left(T_{2}\right)\right\} \\
=\{3 c+2 q+2,3 c+2 q+3, \ldots, 3 c+3 q+1\} .
\end{gathered}
$$

The edge labels form the set of consecutive integers from $c$ up to $3 c+3 q+1$ if and only if $c+3 q+1=3 c+2 q+2$. It is true if $c=(q-1) / 2$. Thus, the labeling $g$ is strongly $(q-1) / 2$ harmonious labeling of the corona graph $K_{2} \odot G$.

An example of the strongly 4-harmonious unicyclic graph is presented in Figure 2.

We know that every odd cycle $C_{2 n+1}$ admits strongly $n$ harmonious labeling. As consequence of Theorem 8 , we have the following.

Corollary 9. The corona graph $K_{2} \odot C_{2 n+1}, n \geq 1$, is strongly n-harmonious.

\section{Conflict of Interests}

The authors declare that there is no conflict of interests regarding the publication of this paper. 


\section{Acknowledgments}

The authors would like to thank the anonymous referees for their valuable comments and suggestions leading to improvement of this paper. The research for this paper was supported by Slovak VEGA Grant 1/0130/12.

\section{References}

[1] R. L. Graham and N. J. A. Sloane, "On additive bases and harmonious graphs," SIAM Journal on Algebraic and Discrete Methods, vol. 1, no. 4, pp. 382-404, 1980.

[2] G. J. Chang, D. F. Hsu, and D. G. Rogers, "Additive variations on a graceful theme: some results on harmonious and other related graphs," Congressus Numerantium, vol. 32, pp. 181-197, 1981.

[3] T. Grace, Graceful, harmonious, and sequential graphs [Ph.D. thesis], University Illinois at Chicago, 1982.

[4] T. Grace, "On sequential labelings of graphs," Journal of Graph Theory, vol. 7, no. 2, pp. 195-201, 1983.

[5] J. A. Gallian, "A dynamic survey graph labeling," The Electronic Journal of Combinatorics, vol. 19, pp. 1-260, 2012.

[6] H. Ullah, G. Ali, M. Ali, and A. Semaničová-Feňovčíková, “On super $(a, d)$-edge-antimagic total labeling of special types of crown graphs," Journal of Applied Mathematics, vol. 2013, Article ID 896815, 6 pages, 2013.

[7] R. Frucht and F. Harary, "On the corona of two graphs," Aequationes Mathematicae, vol. 4, pp. 322-325, 1970.

[8] B. L. Liu and X. K. Zhang, "On a conjecture of harmonious graph," Systems Science and Mathematical Sciences, vol. 2, no. 4, pp. 325-328, 1989.

[9] Y. J. Liu, “The crown graphs $Q_{2 n}$ are harmonious graphs," Hunan Annals of Mathematics, vol. 16, no. 1, pp. 125-128, 1996.

[10] G. S. Singh, "A note on sequential crowns," National Academy Science Letters, vol. 16, no. 9-10, pp. 243-245, 1993.

[11] G. S. Singh, "A note on labeling of graphs," Graphs and Combinatorics, vol. 14, no. 2, pp. 201-207, 1998.

[12] G. Santhosh, "Sequential coronations of graphs," National Academy Science Letters, vol. 28, pp. 269-270, 2005.

[13] S. C. Shee, "On harmonious and related graphs," Ars Combinatoria, vol. 23, pp. 237-247, 1987.

[14] R. B. Gnanajothi, Topics in graph theory [Ph.D. thesis], Madurai Kamaraj University, 1991. 


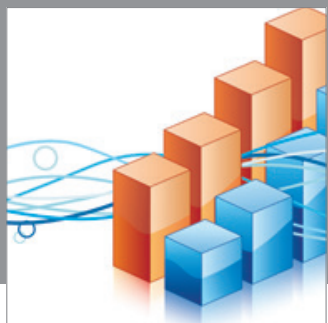

Advances in

Operations Research

mansans

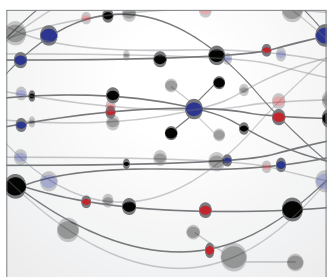

The Scientific World Journal
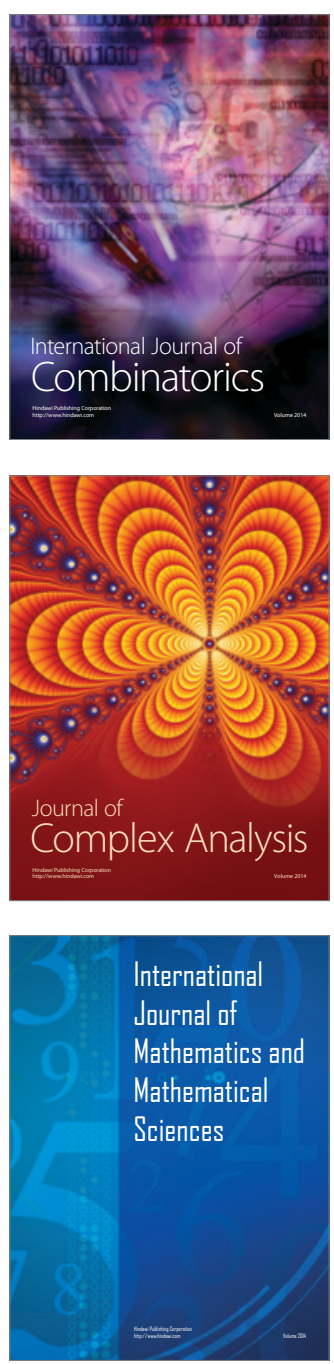
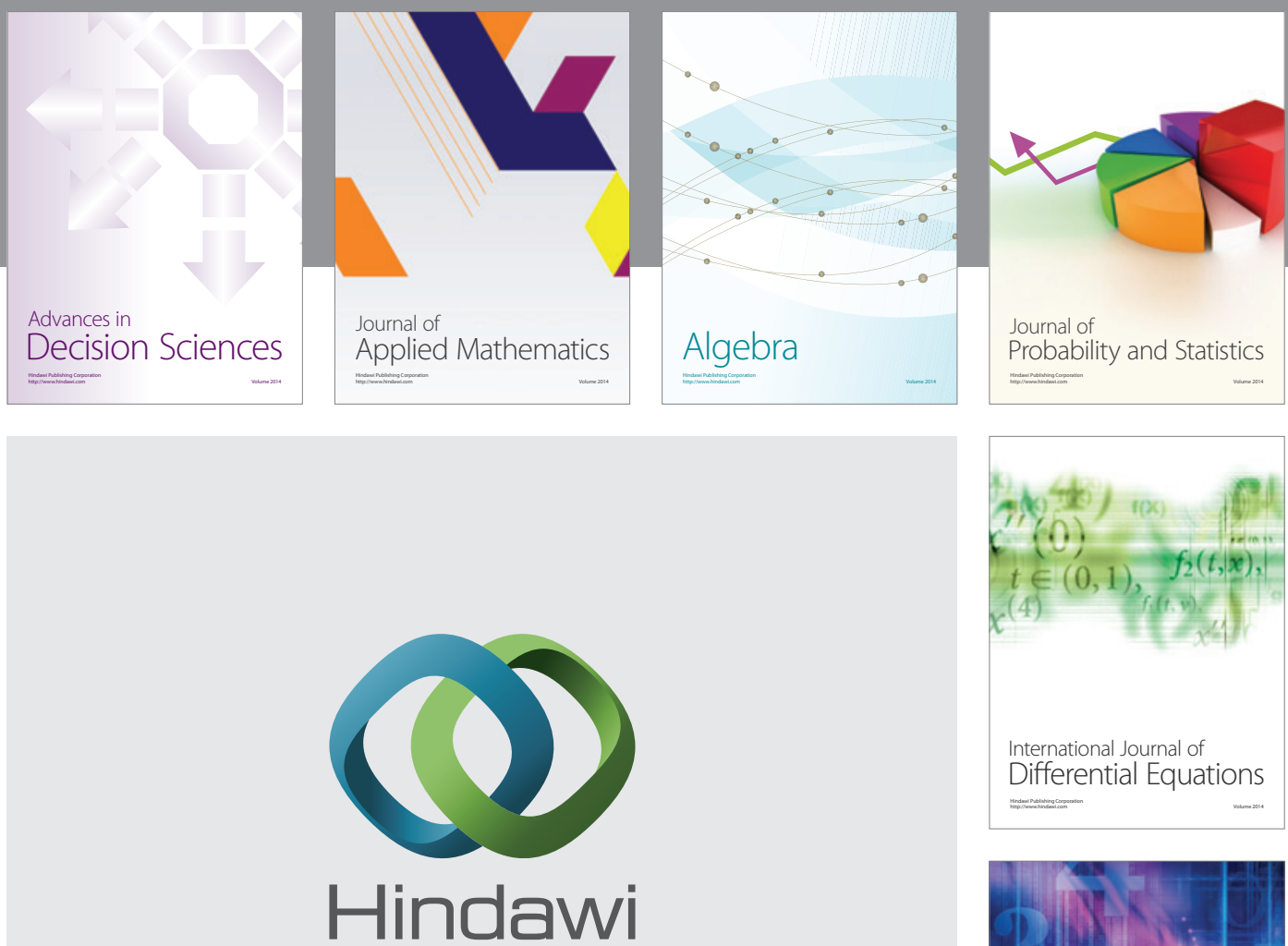

Submit your manuscripts at http://www.hindawi.com
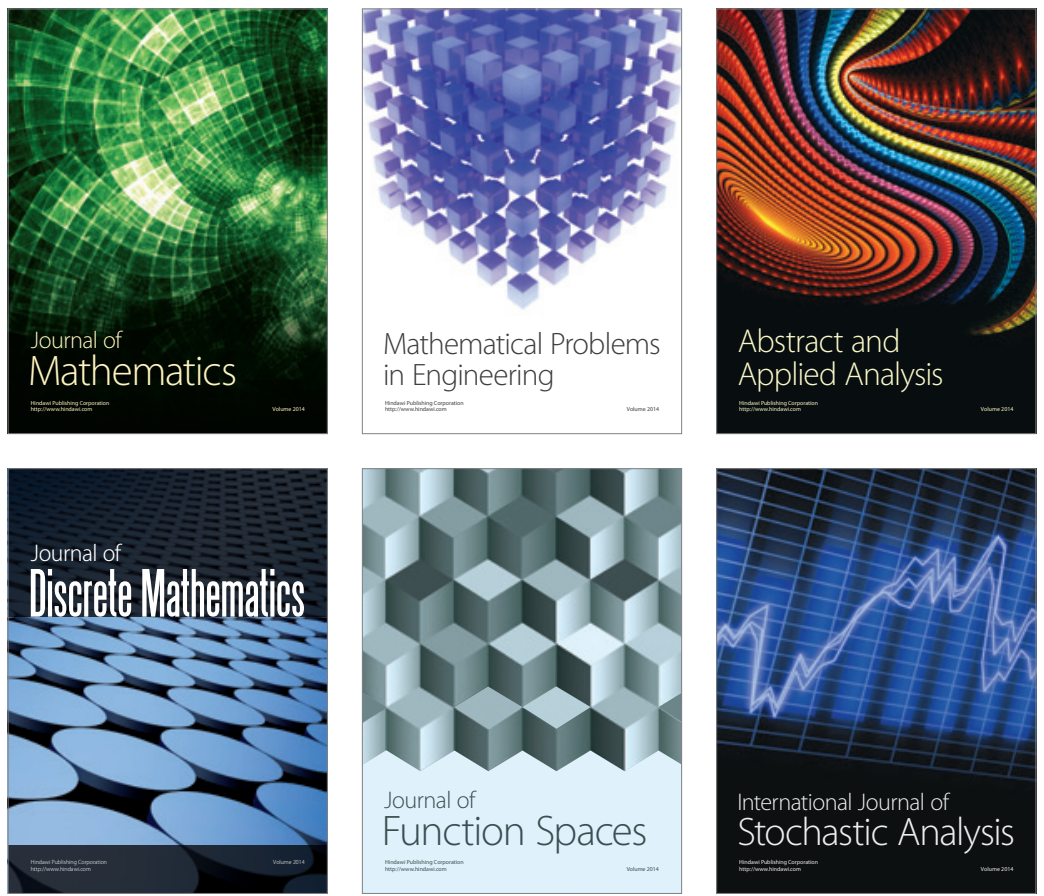

Journal of

Function Spaces

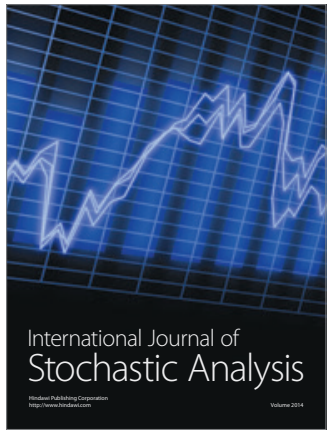

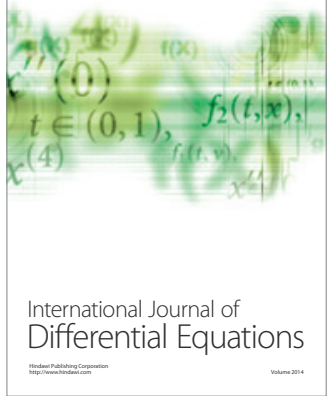
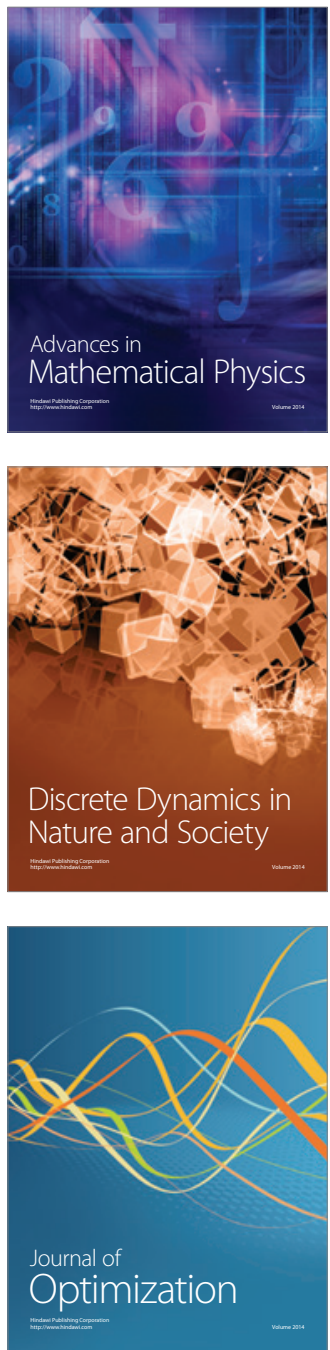